\title{
Detection and Prediction of Absence Seizures Based on Nonlinear Analysis of the EEG in Wag/Rij Animal Model
}

\author{
Saleh Lashkari ${ }^{1}$, Ali Sheikhani ${ }^{*}$, Mohammad Reza Hashemi Golpayegani ${ }^{2}$, Ali Moghimi ${ }^{3}$, Hamidreza Kobravi ${ }^{4}$ \\ 'Department of Biomedical Engineering, Science and Research Branch, Islamic Azad University, Tehran, Iran \\ ${ }^{2}$ Department of Biomedical Engineering, Amirkabir University of Technology, Tehran, Iran \\ ${ }^{3}$ Rayan Center for Neuroscience \& Behavior, Department of Biology, Faculty of Science, Ferdowsi University of Mashhad, \\ Mashhad, Iran \\ ${ }^{4}$ Biomedical Engineering Research Center, Mashhad Branch, Islamic Azad University, Mashhad, Iran
}

\begin{abstract}
Background: Epilepsy is a common neurological disorder with a prevalence of $1 \%$ of the world population. Absence epilepsy is a form of generalized seizures with Spike wave discharge in EEG. Epileptic patients have frequent absence seizures that cause immediate loss of consciousness. Methods: In this study, it has been tried to explore whether EEG changes can effectively detect epilepsy in animal model applying non-linear features. To predict the occurrence of absence epilepsy, a long-term EEG signal has been recorded from frontal cortex in seven Wag/Rij rats. After preprocessing, the data was transferred to the phase space to extract the brain system dynamic and geometric properties of this space. Finally, the ability of each features to predict and detect absence epilepsy with two criteria of predictive time and the accuracy of detection and its results were compared with previous studies.

Results: The results indicate that the brain system dynamic changes during the transition from freeseizure to pre-seizure and then seizure. Proposed approach diagnostic characteristics yielded $97 \%$ accuracy of absence epilepsy diagnosis indicating that due to the nonlinear and complex nature of the system and the brain signal, the use of methods consistent with this nature is important in understanding the dynamic transfer between different epileptic seizures.

Conclusion: By changing the state of the absence Seizures, the dynamics are changing, and the results of this research can be useful in real-time applications such as predicting epileptic seizures. Keywords: Component; Absence epilepsy, Electroencephalogram, Phase space, Nonlinear attractor, Geometric properties
\end{abstract}

*Correspondence to Ali Sheikhani, Department of Biomedical Engineering, Amirkabir University of Technology, Tehran, Iran. Email: sheikhani al $81 @$ srbiau.ac.ir

Published online March 15, 2018

Citation: Lashkari S, Sheikhani A, Hashemi Golpayegani MR, Moghimi A, Kobravi H. Detection and prediction of absence seizures based on nonlinear analysis of the EEG in Wag/Rij animal model. Int Clin Neurosci J. 2018;5(1):21-27. doi: 10.15171/icnj.2018.05.

\section{Introduction}

Epilepsy is one of the most common neurological disorders with a prevalence of $1 \%$ of the world's population. About $80 \%$ of people with epilepsy live in developing countries. ${ }^{1}$ Today, people with epilepsy and their families suffer from social discrimination and social stigma in many parts of the world. Epileptic seizures can be associated with impairment or loss of consciousness. ${ }^{1,2}$

Absence seizures are a form of generalized seizures with Spike wave discharge (SWD) in EEG. ${ }^{2}$ These rapid and sudden seizures are transient symptoms and/or signs of abnormal, excessive or synchronous neuronal activity in the brain. ${ }^{3}$ People with absence epilepsy have repeated seizures that cause momentary lapses of consciousness. ${ }^{4}$ The period of short-lived absence seizures usually lasts from several seconds to about a minute, and may be repeated more than 100 times a day. ${ }^{5}$

Since these sudden and rapid seizures often occur in childhood or adolescence, and may have significant impact on the educational development of patients. ${ }^{6,7}$ Therefore, understanding the transition of brain activity to the absence seizure, called the pre-seizure, is a very difficult goal and is still under discussion., ${ }^{8,9}$

The EEG, which records spontaneous electrical activity in the brain, was first measured in humans by Hans Berger in 1929. Since then, EEG is one of the most useful tools for studying cognitive processes and brain physiopathology, ${ }^{9,10}$ especially the processes involved in epileptic seizures. ${ }^{11,12}$

In the recent decade, dynamics from free-seizure to seizure status has been investigated with different linear or nonlinear methods. ${ }^{10-12}$ To some extent, these results 
showed that the change in EEG features during the seizure could be detected several minutes before the onset of a seizure in focal epilepsy. ${ }^{13-15}$ However, the prediction of sudden and rapid seizures with dynamic EEG changes in people with absence epilepsy is still open problem. ${ }^{16,17}$ Additionally, understanding the transition of brain activity to epileptic seizures and the identification of some pioneering tasks is challenging. ${ }^{8,9,18}$

Sitnikova and van Luijtelaar et al showed that SWD activity in albino rats was associated with the precursor delta and theta short activities in cortex and thalamus. ${ }^{18-20}$ These EEG precursors in rat models provide clues for the prediction of human absence epilepsy. Signal analysis and its trajectory in the phase space can lead to a better understanding of the system's dynamics and provide valuable information about attractors and system behavior. In particular, nonlinear time series analysis methods are presented to identify epileptic seizure states. ${ }^{16,21-26}$ To a certain extent, these methods mainly include the Lyapunov exponent and the correlation dimension, which is able to extract the properties of the useful EEG data to provide evidence to confirm the existence of a previous state of seizure in temporal lobe epilepsy. ${ }^{21-24}$

Ouyang et al used recurrent quantification analysis to distinguish between states in GEARS genetic model of absence epilepsy and show that certainty in seizure periods is higher than in two other states. ${ }^{27}$ Multiscale permutation entropy (MPE) was used to describe the dynamic properties of EEG on various human absence epilepsy and the ability to classify MPE by linear discrimination analysis (LDA) was evaluated. Comparison with the conventional entropy methods with a classification accuracy of $86.1 \%$, the classification rate was $90 \%$ with a MPE index. ${ }^{28}$ Similarly, in Li et al study using permutation entropy, an examination was implemented to determine whether EEG data changes can detect various states of human absence epilepsy. ${ }^{1}$ The average PE values have been shown to gradually decrease from no seizure to seizure state and provide evidence that three different phases of seizure in absence epilepsy can be detectable.

Despite numerous studies conducted on human EEG and animal models, it has been largely attributed to the extraction of global features. The evolutionary characteristics of the EEG signal based on the dynamics of turbulence is still an open issue. ${ }^{27}$ Using geometric features, regardless of any assumptions about the type of dynamics of the basis, provides studies independent of the hypothesis of chaos and therefore independent of the analysis of alternative data. ${ }^{29}$ This study attempts to apply features based on phase space geometry to discover whether EEG changes can effectively identify absence seizures.

\section{Materials and Methods}

Surgery and Recording System

EEG epochs were obtained from 7 of genetic absence

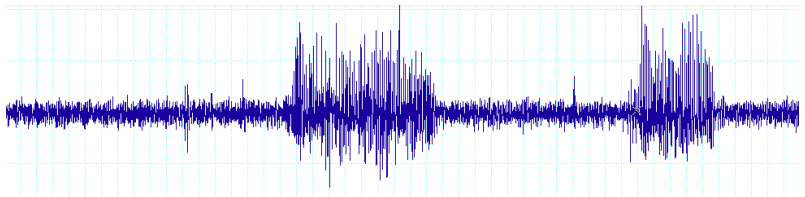

Figure 1. EEG Segment of Absence Seizure With Spike Wave Discharge.

epilepsy Wag/Rij rats (Male, weight $300 \pm 5$ g) with a minimum age of 5 months. Animals were anesthetized with ketamine $100 \mathrm{mg} / \mathrm{kg}$ and xylazine $10 \mathrm{mg} / \mathrm{kg}$. In all animals, dipole EEG stainless steel electrodes were placed in the frontal cortex area $(1 \mathrm{~mm}$ above Bregma and 3 $\mathrm{mm}$ from Lambda) and the reference electrode was also placed in the temporal region. Each animal was kept in a separate box for recovery in the animal house. The EEG electrode signal was directly connected to the BioAmp ML 136 amplifier and stored using LabChart software. The EEG data was recorded for a long term of 48 to 72 hours at a sampling rate of $1 \mathrm{kHz}$ using a 16-bit ADC and a filter with frequency band of $0.5-100 \mathrm{~Hz}$ (Figure 1).

\section{Feature Extraction}

Based on the well-known theory of phase space and embedding, Takens states that system behavior in state space can be estimated by a vector of observation, such as EEG. A variety of new concepts and time series analysis techniques have been developed to allow the description of the behavioral dynamics of the system for an indefinite system. Proposed geometric properties can be calculated in accordance with equation (1) to the trajectory $x(t)$ in dimension $\mathrm{d}$. In practice, all $d$ states are not accessible in the phase space, and only the vector of observations $x(t)$ is available. ${ }^{30}$

$\vec{x}(t)=\left[x_{1}(t), x_{2}(t), \ldots, x_{d}(t)\right]$

The Takens method, ${ }^{19}$ is used frequently to embedded the time series $x(t)$ into the d-dimensional phase space:

$\vec{x}(t)=[x(t), x(t-\tau) \ldots x(t-(d-1) \tau)]$

Where $d$ is the embedding dimension and $\tau$ is the lag estimated by the false nearest neighbor algorithm ${ }^{31}$ and the mean of mutual information. ${ }^{32}$ Table 1 lists the features list, some of which are presented below.

\section{Center of Gravity}

The center $\left(\mathrm{g}_{\mathrm{x}}, \mathrm{g}_{\mathrm{y}}\right)$ is equal to:

$\left\{\begin{array}{l}g_{x}=\frac{1}{N} \sum_{i=1}^{N} x_{i} \\ g_{y}=\frac{1}{N} \sum_{i=1}^{N} y_{i}\end{array}\right.$

Where $N$ is the number of points in the 2-D phase space. 
Table 1. Geometric Features of Phase Space ${ }^{33}$

\begin{tabular}{lll}
\hline Feature & Symbol & Description \\
\hline 1 & $\mathrm{~A}$ & Area \\
2 & $\mathrm{G}_{\mathrm{x}}$ & Horizontal center of gravity \\
3 & $\mathrm{G}_{\mathrm{y}}$ & Vertical center of gravity \\
4 & $\mathrm{I}$ & Axis of Least Inertia \\
5 & $\mathrm{E}$ & Eccentricity \\
6 & $\mathrm{C}_{\mathrm{va}}$ & Circularity ratio \\
7 & $\mathrm{C}_{\mathrm{va}}$ & Ellipse variance \\
8 & $\mathrm{~B}$ & Average bending energy \\
9 & $\mathrm{P}$ & Perimeter \\
10 & $\mathrm{R}$ & Rectangularity \\
11 & $\mathrm{TAR}$ & Triangle area representation \\
12 & $\mathrm{CD}$ & Centroid distance \\
13 & $\mathrm{CC}$ & Complex coordinate \\
\hline
\end{tabular}

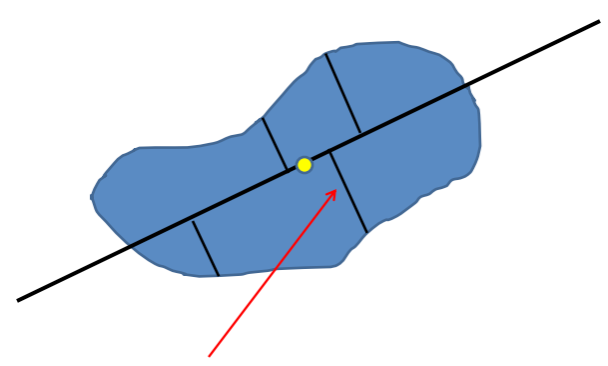

Figure 2. Least Inertia. The yellow point shows the center of gravity. ${ }^{33}$

\section{Axis of Least Inertia}

The least inertia axis, the $A L I$, is used as a unique reference line to maintain the shape orientation, and is defined in such a way that the square integral of the distances from the boundary points is minimal (Figure 2).

\section{Average Bending Energy}

The average bending energy, $\mathrm{BE}$, is defined as:

$$
B E=\frac{1}{N} \sum_{s=0}^{N-1} K(s)^{2}
$$

$K(s)$ is the Curvature function, $s$ is the parameter of the arc length and $N$ is the number of contour points. ${ }^{34}$

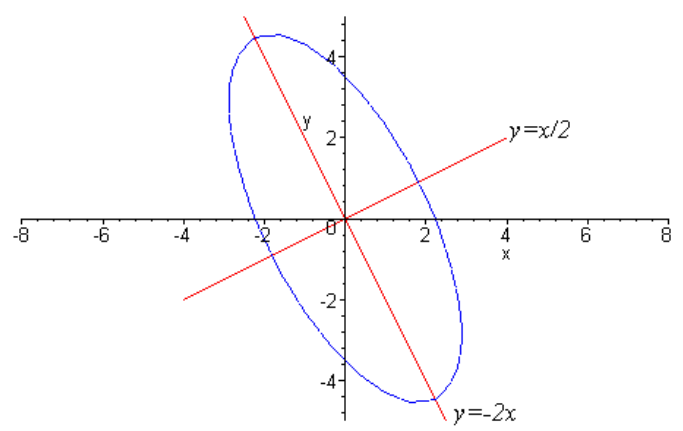

Figure 3. Centrifugal Centered Ellipse. ${ }^{35}$

\section{Eccentricity}

This criterion measures the aspect ratio, and in fact the ratio of the axis to the small one (Figure 3). It can be calculated using the principal axis method or the minimum bounding rectangle method. ${ }^{33}$

\section{Circularity Ratio}

The circularity ratio explains how the shape is similar to a circle ${ }^{35}$ :

$\mathrm{C}_{\mathrm{va}}=\frac{\sigma_{\mathrm{R}}}{\mu_{\mathrm{R}}}$

$\mu$ and $\sigma$ are the mean and standard deviation of the radial distance from the center of gravity of the basin of attraction relative to the boundary points.

\section{Ellipse Variance}

This criterion is equivalent to mapping error to fit to the ellipse with a similar covariance matrix. ${ }^{33}$

\section{Rectangularity}

The rectangularity states how and to what extent the minimum rectangle fills the boundary:

Rectangularity $=\frac{A_{S}}{A_{R}}$

Where $A_{S}$ is the area of the shape and $A_{R}$, the area of the minimum rectangle is the range..$^{33}$

\section{Convexity}

The ratio of the perimeter of the convex cortex to the contour environment ${ }^{36}$ :

$$
\text { Convexity }=\frac{\mathrm{O}_{\text {Convexhull }}}{0}
$$

\section{Complex Coordinate}

The complex coordinate function is the simplification of complex numbers generated from the coordinates of the boundary points ${ }^{33}$ :

$$
\left\{\begin{array}{cc}
P_{n}(x(n), y(n)), \quad n \in[1, N] \\
z(n)=\left[x(n)-g_{x}\right]+i\left[y(n)-g_{y}\right]
\end{array}\right.
$$

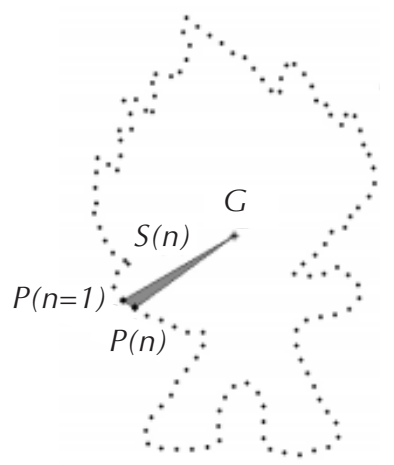

Figure 4. Area function, $S(n)$ is the area between the boundary points $P_{n^{\prime}} P_{n+1}$ and the center of gravity, G. ${ }^{33}$ 


\section{Area Function}

By changing the boundary points along the boundary, the area of the triangle formed with two border points and the center of gravity also changes (Figure 4). ${ }^{33}$

\section{Triangle Area Representation}

The triangle area representation, TAR, is calculated by the area of the triangle formed by points on the boundary of

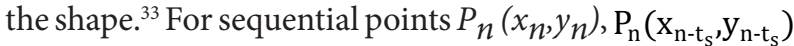
and $P_{n}\left(\mathrm{x}_{\mathrm{n}+\mathrm{t}_{\mathrm{s}}}, \mathrm{y}_{\mathrm{n}+\mathrm{t}_{\mathrm{s}}}\right), N$ is even.

$\operatorname{TAR}\left(n, t_{s}\right)=\frac{1}{2}\left|\begin{array}{lll}x_{n-t_{s}} & y_{n-t_{s}} & 1 \\ x_{n} & y_{n} & 1 \\ x_{n+t_{s}} & y_{n+t_{s}} & 1\end{array}\right|$

\section{Feature Evaluation}

Chaotic a $\mathrm{n} \mathrm{d}$ nonlinear dynamics features have two important characteristics:

- is sensitive to the dynamic changes of the system.

- is invariant to the initial conditions change.

So, firstly, the proposed features were evaluated with the Lorenz system trajectory, Equation 10.

Lorenz system: $\left\{\begin{array}{c}\frac{d X}{d t}=\sigma(Y-Z) \\ \frac{d Y}{d t}=r X-Y-X Z \\ \frac{d Z}{d t}=X Y-\beta Z\end{array}\right.$

To evaluate the features, each was calculated for different parameters of Lorenz system and 10 iterations. If the distribution is different from each other for different values of the parameter, the feature will be invariant. This significant separability is tested by two-sample $t$ test method (Figure 5).

\section{Results}

Table 2 represents the $P$ values of the Jarque-Bera test for feature values for each of the parameters. The values of $P>0.05$ show that the values of features follow the normal distribution. As it can be seen in most cases the p-value is greater than 0.05 , so that the $t$ test can be applied to the values of the features.

Figure 6 present results of two-sample $t$ test at the 5\% significance level. Each black pixel shows feature values that causes pixels are independent. This means that the two sets of parameter values are meaningfully separated. It is observed that the properties of the Lorenz system are highly sensitive to parameter variations and are invariant due to initial conditions variations. After evaluating the features, phase space analysis and their calculation on the EEG signal of the animal model were made. As it can be seen, the geometry of the phase space of the EEG in the transition from seizure-free to seizure state is changing (Figure 7). The phase space symmetry is lost in transit from seizure free to seizure state, in such a way that the phase space has become asymmetrical from the ellipse shape. Additionally, the contraction and expansion in the phase space of the seizure state is the signs of the stretching

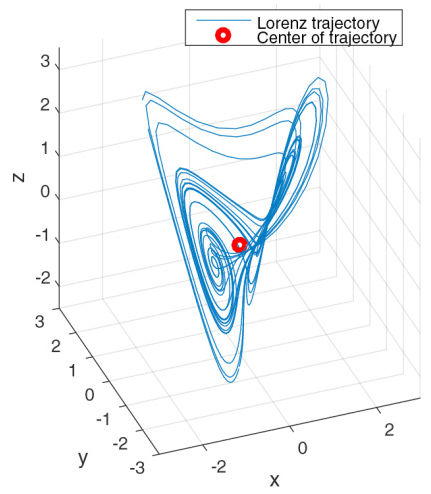

Figure 5. The Trajectory of the Lorenz System for $\sigma=16, r=50$ and $\beta=4$.

Table 2. The P Values of Features Values for Normality Test

\begin{tabular}{lccccc}
\hline Feature & \multicolumn{5}{c}{ Lorenz System } \\
\hline $\mathrm{A}$ & $\mathbf{0 . 5 0}$ & $\mathbf{0 . 5 0}$ & 0.01 & $\mathbf{0 . 3 0}$ & $\mathbf{0 . 1 3}$ \\
$\mathrm{G}_{\text {x }}$ & $\mathbf{0 . 2 7}$ & $\mathbf{0 . 5 0}$ & $\mathbf{0 . 5 0}$ & $\mathbf{0 . 1 8}$ & $\mathbf{0 . 2 0}$ \\
$\mathrm{G}_{y}$ & $\mathbf{0 . 4 2}$ & $\mathbf{0 . 2 1}$ & $\mathbf{0 . 0 7}$ & 0.005 & $\mathbf{0 . 5 0}$ \\
$\mathrm{I}$ & $\mathbf{0 . 2 3}$ & $\mathbf{0 . 5 0}$ & $\mathbf{0 . 2 7}$ & $\mathbf{0 . 4 1}$ & $\mathbf{0 . 5 0}$ \\
$\mathrm{E}$ & $\mathbf{0 . 5 0}$ & $\mathbf{0 . 0 7}$ & $\mathbf{0 . 3 5}$ & $\mathbf{0 . 0 5}$ & $\mathbf{0 . 5 0}$ \\
$\mathrm{C}_{\text {va }}$ & 0.002 & $\mathbf{0 . 5 0}$ & 0.01 & $\mathbf{0 . 3 3}$ & $\mathbf{0 . 5 0}$ \\
$\mathrm{E}$ & $\mathbf{0 . 5 0}$ & $\mathbf{0 . 0 7}$ & $\mathbf{0 . 3 5}$ & $\mathbf{0 . 0 5}$ & $\mathbf{0 . 5 0}$ \\
$\mathrm{E}_{\text {va }}$ & $\mathbf{0 . 2}$ & $\mathbf{0 . 5 0}$ & $\mathbf{0 . 2 8}$ & $\mathbf{0 . 3}$ & $\mathbf{0 . 1}$ \\
$\mathrm{R}$ & $\mathbf{0 . 5 0}$ & 0.004 & $\mathbf{0 . 5 0}$ & $\mathbf{0 . 1 4}$ & $\mathbf{0 . 3 7}$ \\
TAR & $\mathbf{0 . 5 0}$ & $\mathbf{0 . 5 0}$ & $\mathbf{0 . 3 4}$ & $\mathbf{0 . 1 1}$ & $\mathbf{0 . 1}$ \\
CD & 0.004 & $\mathbf{0 . 5 0}$ & $\mathbf{0 . 3 7}$ & $\mathbf{0 . 1 7}$ & 0.001 \\
CC & $\mathbf{0 . 4 0}$ & $\mathbf{0 . 2 1}$ & 0.002 & $\mathbf{0 . 5 0}$ & $\mathbf{0 . 5 0}$ \\
\hline
\end{tabular}

The bold values are greater than 0.05 that means feature values come from normal distribution.
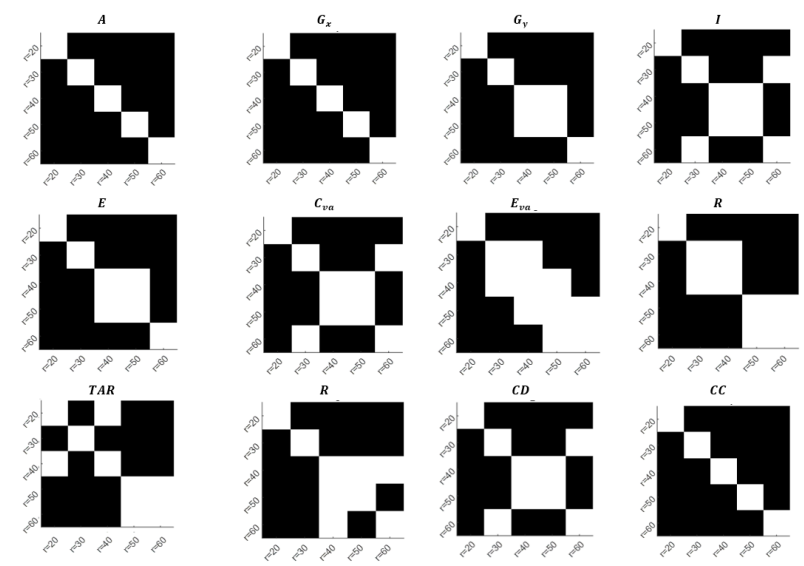

Figure 6. The result of $t$ test between all pair of parameters for each of the features which are extracted from Lorenz trajectories. The black color shows that the feature values for pair parameter are independent.

and folding of the EEG in this state, which confirms the existence of chaotic degrees even in seizure mode.

Figure 8A-E shows values of some of the features for 5 seconds before the seizure and 10 seconds after for 1500 EEG segments. 


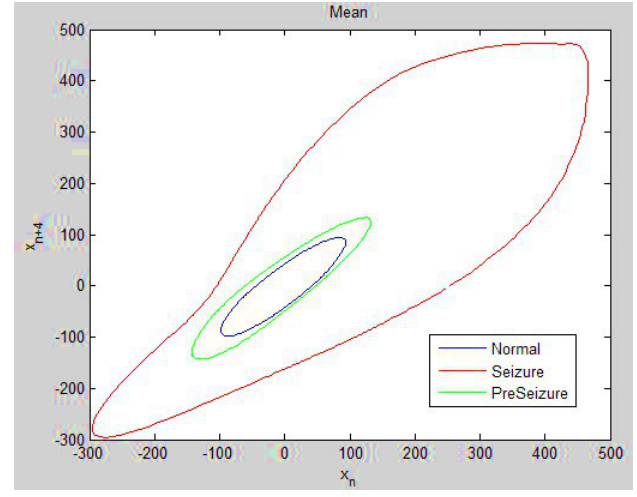

Figure 7. The Mean Phase-Space Contour of the 1500 EEG Segments in an Animal Model for Free-Seizure, Pre-seizure, and Seizure States.

According to the results of the counter area, the phase space in the transition from free-seizure state to a seizure state is increasing (Figure 8A).

In addition to changing the geometry of the phase space, changing the position of the phase space on the phase space can reveal the dynamic changes of the EEG signal, which in this transition the position of the center of gravity of the phase space is being displaced (Figure $8 \mathrm{~B})$. With regard to the significant changes in the Axis of Least Inertia (Figure 8C), it is proved that the orientation of the phase space in the transition to the seizure varies considerably. Other features also showed significant changes (Figures 8C-E).

In order to achieve the optimal threshold, the detection accuracy curve was calculated in terms of threshold for each of the features and the optimal threshold was determined. Figure 9 shows this curve for the Axis of
Table 3. Average Accuracy of Geometric Features for 1500 EEG Segments

\begin{tabular}{ll}
\hline Feature & Mean ACC \% \\
\hline$A$ & 97 \\
$G_{x}$ & 91 \\
$G_{y}$ & 82.5 \\
I & 97 \\
$E$ & 25 \\
$C_{v a}$ & 66 \\
$E_{v a}$ & 64 \\
$B$ & 81 \\
$P$ & 95.5 \\
$R$ & 47 \\
TAR & 10.5 \\
$C D$ & 97 \\
$C C$ & 94 \\
\hline
\end{tabular}

\section{Least Inertia feature.}

\section{Discussion}

In this Research, it was attempted to determine whether EEG changes could effectively identify the free-seizure, pre-seizure and seizure states in the animal model of absence epilepsy by applying features based on phasespace geometry (Table 3). Unlike traditional time series analysis, nonlinear dynamics describes the nonlinear relation of system variables by examining variables only in phase space. The main strength of this method is to provide information in accordance with the basic dynamics of the system without having information of all the evolution parameters in the system. Hence, in this approach, the dynamics of the system in the reconstruction phase space
(A)

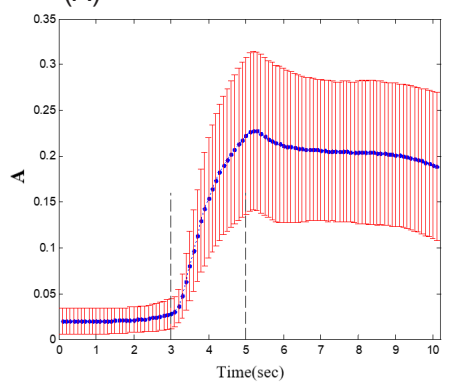

(D)

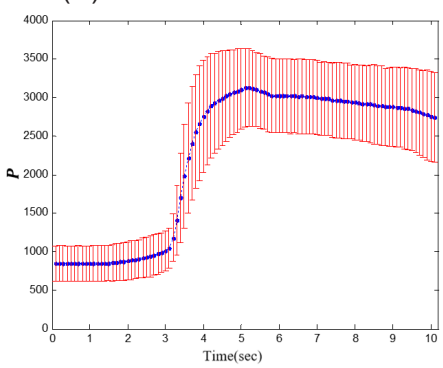

$(B)$

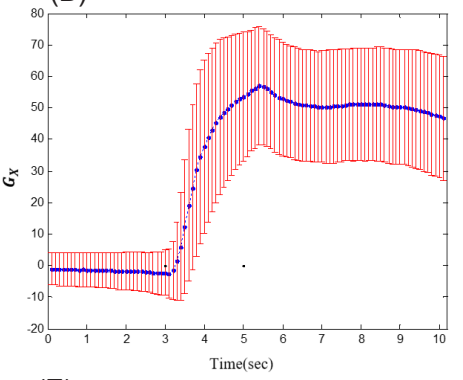

(E)

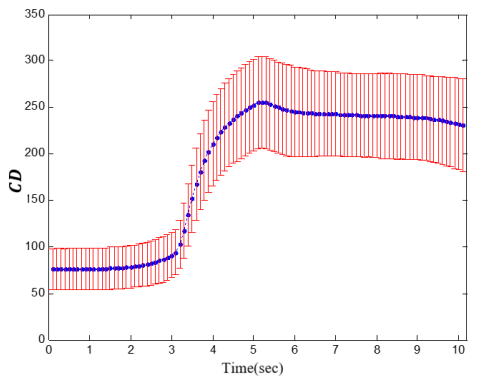

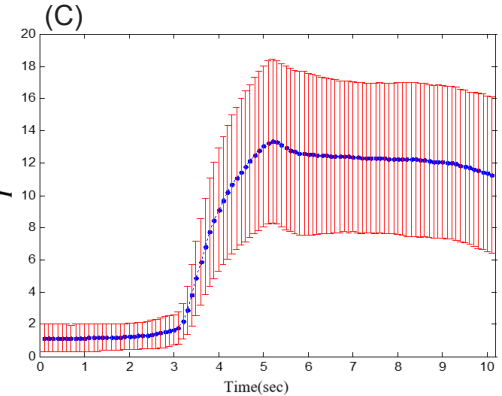

Figure 8. (A) Representation of Area feature, A. (B) Horizontal Center of Gravity, $G_{x}$. (C) Axis of Least Inertia, I. (D) Perimeter, P. (E) Centroid Distance, $C D$. 


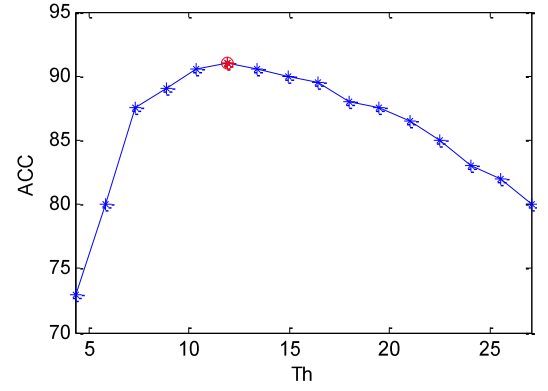

Figure 9. Determination of the Optimal Threshold by Calculating the Seizure Detection Accuracy for Different Thresholds.

Table 4. Performance comparison with some existing methods that use the same data sets

\begin{tabular}{llll}
\hline ACC\% & Seizures & Year & Reference \\
\hline $21 \& 54$ & 314 & 2007 & 16 \\
94 & Not reported & 2008 & 37 \\
Accuracy higher than 90 & 202 & 2009 & 38 \\
85 & Not reported & 2009 & 39 \\
94.8 & 73 & 2014 & 40 \\
91.8 & & & \\
94.9 & 205 & 2015 & 41 \\
89.9 & & \\
For three absence genes & & & \\
\hline
\end{tabular}

and then the reconstructed attractors were quantified.

Table 4 compares the results of the proposed approach and previous studies in the detection of absence seizures. As the results suggest, the performance of the proposed method has improved in comparison with other previous studies. One of the highlights of this study is generalization, so that 1500 segments were used for evaluation, however, with a $97 \%$ accuracy.

In addition, in previous studies, it has often used linear approaches to quantification of the EEG signal, while the nature of the EEG signal is particularly nonlinear and it is necessary to provide an approach to detect the dynamics of this signal. The shape clearly confirms the appropriateness of the dynamic variation of the phase space with the transition of the EEG signal from freeseizure to the seizure phase.

In this study, it was shown that simple, yet meaningful features such as the area and the environment can be found to provide best detection. Certainly, the application of more complex pattern identification systems will improve the detection results. Dynamic changes during multiple seizures and the prognosis of the disease are among the issues discussed in examining absence epilepsy with the use of the EEG signal.

\section{Conflict of Interest Disclosures}

The authors declare that they have no conflict of interests.

\section{Ethical Statement}

All animal experiments were conducted in compliance with the international ethical and animal-laboratory regulations and the Code of Ethics for Laboratory and also according to Human and animal research ethics committee rules of Ferdowsi University of

Mashhad.

\section{References}

1. Li J, Yan J, Liu X, Ouyang G. Using Permutation Entropy to Measure the Changes in EEG Signals During Absence Seizures. Entropy. 2014;16(6):3049-61. doi: 10.3390/e16063049.

2. Gorji A, Mittag C, Shahabi P, Seidenbecher T, Pape HC. Seizurerelated activity of intralaminar thalamic neurons in a genetic model of absence epilepsy. Neurobiol Dis. 2011;43(1):266-74. doi: 10.1016/j.nbd.2011.03.019.

3. Meeren HK, Pijn JP, Van Luijtelaar EL, Coenen AM, Lopes da Silva FH. Cortical focus drives widespread corticothalamic networks during spontaneous absence seizures in rats. J Neurosci. 2002;22(4):1480-95.

4. Cavanna AE, Monaco F. Brain mechanisms of altered conscious states during epileptic seizures. Nat Rev Neurol. 2009;5(5):26776. doi: 10.1038/nrneurol.2009.38.

5. Bai X, Vestal M, Berman R, Negishi M, Spann M, Vega C, et al. Dynamic time course of typical childhood absence seizures: EEG, behavior, and functional magnetic resonance imaging. J Neurosci. 2010;30(17):5884-93. doi: 10.1523/ jneurosci.5101-09.2010.

6. Killory BD, Bai X, Negishi M, Vega C, Spann MN, Vestal M, et al. Impaired attention and network connectivity in childhood absence epilepsy. Neuroimage. 2011;56(4):2209-17. doi: 10.1016/j.neuroimage.2011.03.036.

7. Blumenfeld H. Impaired consciousness in epilepsy. Lancet Neurol. 2012;11(9):814-26. doi: 10.1016/s14744422(12)70188-6.

8. Rosso OA, Mendes A, Berretta R, Rostas JA, Hunter M, Moscato P. Distinguishing childhood absence epilepsy patients from controls by the analysis of their background brain electrical activity (II): a combinatorial optimization approach for electrode selection. J Neurosci Methods. 2009;181(2):257-67. doi: 10.1016/j.jneumeth.2009.04.028.

9. Crunelli V, Cope DW, Terry JR. Transition to absence seizures and the role of GABA(A) receptors. Epilepsy Res. 2011;97(3):283-9. doi: 10.1016/j.eplepsyres.2011.07.011.

10. Kramer MA, Eden UT, Kolaczyk ED, Zepeda R, Eskandar EN, Cash SS. Coalescence and fragmentation of cortical networks during focal seizures. J Neurosci. 2010;30(30):10076-85. doi: 10.1523/jneurosci.6309-09.2010.

11. Neymotin SA, Lee H, Fenton AA, Lytton WW. Interictal EEG discoordination in a rat seizure model. J Clin Neurophysiol. 2010;27(6):438-44. doi: 10.1097/WNP.0b013e3181fe059e.

12. Schindler $\mathrm{K}$, Leung $\mathrm{H}$, Elger $\mathrm{CE}$, Lehnertz $\mathrm{K}$. Assessing seizure dynamics by analysing the correlation structure of multichannel intracranial EEG. Brain. 2007;130(Pt 1):65-77. doi: 10.1093/ brain/awl304.

13. Mormann F, Elger CE, Lehnertz K. Seizure anticipation: from algorithms to clinical practice. Curr Opin Neurol. 2006;19(2):187-93. doi: 10.1097/01.wco.0000218237.52593. bc.

14. Mormann F, Andrzejak RG, Elger CE, Lehnertz K. Seizure prediction: the long and winding road. Brain. 2007;130(Pt 2):314-33. doi: 10.1093/brain/awl241.

15. Stacey W, Le Van Quyen M, Mormann F, Schulze-Bonhage A. What is the present-day EEG evidence for a preictal state? Epilepsy Res. 2011;97(3):243-51. doi: 10.1016/j. eplepsyres.2011.07.012.

16. Li X, Ouyang G, Richards DA. Predictability analysis of absence seizures with permutation entropy. Epilepsy Res. 2007;77(1):704. doi: 10.1016/j.eplepsyres.2007.08.002.

17. Stacey WC, Litt B. Technology insight: neuroengineering and epilepsy-designing devices for seizure control. Nat Clin Pract Neurol. 2008:4(4):190-201. doi: 10.1038/ncpneuro0750.

18. Sitnikova E. Thalamo-cortical mechanisms of sleep spindles and spike-wave discharges in rat model of absence epilepsy 
(a review). Epilepsy Res. 2010;89(1):17-26. doi: 10.1016/j. eplepsyres.2009.09.005.

19. Sitnikova E, van Luijtelaar G. Electroencephalographic precursors of spike-wave discharges in a genetic rat model of absence epilepsy: Power spectrum and coherence EEC analyses. Epilepsy Res. 2009;84(2-3):159-71. doi: 10.1016/j. eplepsyres.2009.01.016.

20. van Luijtelaar G, Hramov A, Sitnikova E, Koronovskii A. Spikewave discharges in WAG/Rij rats are preceded by delta and theta precursor activity in cortex and thalamus. Clin Neurophysiol. 2011;122(4):687-95. doi: 10.1016/j.clinph.2010.10.038.

21. Andrzejak RG, Lehnertz K, Mormann F, Rieke C, David P, Elger CE. Indications of nonlinear deterministic and finitedimensional structures in time series of brain electrical activity: dependence on recording region and brain state. Phys Rev E Stat Nonlin Soft Matter Phys. 2001;64(6 Pt 1):061907. doi: 10.1103/PhysRevE.64.061907.

22. Elger CE, Lehnertz K. Seizure prediction by non-linear time series analysis of brain electrical activity. Eur J Neurosci. 1998;10(2):786-9.

23. lasemidis LD. Epileptic seizure prediction and control. IEEE Trans Biomed Eng. 2003;50(5):549-58

24. Lehnertz K, Elger CE. Can Epileptic Seizures be Predicted? Evidence from Nonlinear Time Series Analysis of Brain Electrical Activity. Phys Rev Lett. 1998;80(22):5019-22. doi: 10.1103/PhysRevLett.80.5019.

25. Winterhalder M, Schelter B, Maiwald T, Brandt A, Schad A, Schulze-Bonhage $A$, et al. Spatio-temporal patient-individual assessment of synchronization changes for epileptic seizure prediction. Clin Neurophysiol. 2006;117(11):2399-413. doi: 10.1016/j.clinph.2006.07.312.

26. Navarro V, Martinerie J, Le Van Quyen M, Clemenceau S, Adam C, Baulac M, et al. Seizure anticipation in human neocortical partial epilepsy. Brain. 2002;125(Pt 3):640-55.

27. Ouyang G, Li X, Dang C, Richards DA. Using recurrence plot for determinism analysis of EEG recordings in genetic absence epilepsy rats. Clin Neurophysiol. 2008;119(8):1747-55. doi: 10.1016/j.clinph.2008.04.005.

28. Ouyang G, Li J, Liu X, Li X. Dynamic characteristics of absence EEG recordings with multiscale permutation entropy analysis. Epilepsy Res. 2013;104(3):246-52. doi: 10.1016/j. eplepsyres.2012.11.003.

29. Zabihi M, Kiranyaz S, Rad AB, Katsaggelos AK, Gabbouj M, Ince T. Analysis of High-Dimensional Phase Space via Poincare Section for Patient-Specific Seizure Detection. IEEE Trans Neural Syst Rehabil Eng. 2016;24(3):386-98. doi: 10.1109/ tnsre.2015.2505238.
30. Lehnertz K. Epilepsy and nonlinear dynamics. J Biol Phys. 2008;34(3-4):253-66. doi: 10.1007/s10867-008-9090-3.

31. Kennel MB, Brown R, Abarbanel HD. Determining embedding dimension for phase-space reconstruction using a geometrical construction. Phys Rev A. 1992;45(6):3403-11.

32. Fraser AM, Swinney HL. Independent coordinates for strange attractors from mutual information. Phys Rev A Gen Phys. 1986;33(2):1134-40.

33. Yang $M$, Kpalma K, Ronsin J. A Survey of Shape Feature Extraction Techniques. In: Peng-Yeng Y, ed. Pattern Recognit. IN-TECH; 2008:43-90.

34. Loncaric S. A survey of shape analysis techniques. Pattern Recognit. 1998;31(8):983-1001. doi: 10.1016/S00312023(97)00122-2

35. Zhang D, Lu G. Review of shape representation and description techniques. Pattern Recognit. 2004;37(1):1-19. doi: 10.1016/j. patcog.2003.07.008.

36. Peura $\mathrm{M}$, livarinen J. Efficiency of simple shape descriptors. In: Arcelli C, Cordella LP, Baja GS, eds. Advances in Visual Form Analysis. Singapore: World Scientific; 1997:443-51.

37. Van Hese P, Martens JP, Waterschoot L, Boon P, Lemahieu I. Automatic detection of spike and wave discharges in the EEG of genetic absence epilepsy rats from Strasbourg. IEEE Trans Biomed Eng. 2009;56(3):706-17. doi: 10.1109/ tbme.2008.2008858.

38. Xanthopoulos P, Liu CC, Zhang J, Miller ER, Nair SP, Uthman $\mathrm{BM}$, et al. A robust spike and wave algorithm for detecting seizures in a genetic absence seizure model. Conf Proc IEEE Eng Med Biol Soc. 2009;2009:2184-7. doi: 10.1109/ iembs.2009.5334941.

39. Buteneers P, Schrauwen B, Verstraeten D, Stroobandt D. RealTime Epileptic Seizure Detection on Intra-cranial Rat Data Using Reservoir Computing. Berlin, Heidelberg: Springer; 2009.

40. Startceva SA, Luettjohann A, Sysoev IV, van Luijtelaar G. A new method for automatic marking epileptic spike-wave discharges in local field potential signals. Proceedings Volume 9448, Saratov Fall Meeting 2014: Optical Technologies in Biophysics and Medicine XVI; Laser Physics and Photonics XVI; and Computational Biophysics; 94481R (2015). doi: $10.1117 / 12.2179017$

41. Richard CD, Tanenbaum A, Audit B, Arneodo A, Khalil A, Frankel WN. SWDreader: a wavelet-based algorithm using spectral phase to characterize spike-wave morphological variation in genetic models of absence epilepsy. J Neurosci Methods. 2015;242:127-40. doi: 10.1016/j.jneumeth.2014.12.016. 